\title{
Plant interactions with microbes and insects: from molecular mechanisms to ecology
}

\author{
Corné M.J. Pieterse ${ }^{1}$ and Marcel Dicke ${ }^{2}$ \\ ${ }^{1}$ Plant-Microbe Interactions, Institute of Environmental Biology, Faculty of Science, Utrecht University, P.O. Box 800.84, 3508 TB \\ Utrecht, The Netherlands \\ ${ }^{2}$ Laboratory of Entomology, Wageningen University, P.O. Box 8031, 6700 EH Wageningen, The Netherlands
}

\begin{abstract}
Plants are members of complex communities and interact both with antagonists and beneficial organisms. An important question in plant defense-signaling research is how plants integrate signals induced by pathogens, beneficial microbes and insects into the most appropriate adaptive response. Molecular and genomic tools are now being used to uncover the complexity of the induced defense signaling networks that have evolved during the arms races between plants and their attackers. Molecular biologists and ecologists are joining forces to place molecular mechanisms of plant defense into an ecological perspective. Here, we review our current understanding of the molecular mechanisms of induced plant defense and their potential ecological relevance in nature.
\end{abstract}

\section{Plants interact with friends and foes}

Plants are abundantly present on Earth and are at the basis of most food webs. Each of the $\sim 300000$ plant species is attacked by a multitude of other organisms, which includes insects and pathogens. For instance, the number of insect species is estimated to be in the order of six million, half of which are herbivorous [1]. The diversity of pathogenic microbes is less well characterized but their threat to plants is equally renowned [2]. In addition to parasitic interactions, beneficial relationships are also frequent in nature, improving plant nutrition or helping the plant to overcome abiotic and biotic stresses. These interactions involve fungi, such as mycorrhizal symbionts; bacteria, such as plant growth-promoting rhizobacteria; natural enemies of attacking insects, such as predators and parasitoids of herbivores that are attracted to volatiles emitted by herbivore-challenged plants; and pollinators, such as moths, ants or bees that are attracted to flower volatiles [3-8] (Figure 1). The establishment of mutualistic associations usually involves mutual recognition and a high degree of coordination at the morphological and physiological level, which requires a continuous cellular and molecular dialogue between the plant and the beneficial organism [3].

To combat effectively invasion by pathogens and insects on the one hand, and to accommodate beneficial organisms

Corresponding author: Dicke, M. (Marcel.Dicke@wur.nl).

Available online 8 November 2007. on the other hand, plants have evolved sophisticated strategies to 'perceive' biotic interactions and to translate this 'perception' into a defensive or conducive response [3,9-12] (Box 1). Recent genomics research has revealed that the capacity of the plant to respond to the enormous diversity of attackers and beneficials is highly flexible [1317]. The signaling networks that are activated by the plant in response to parasitic, herbivorous and beneficial organisms overlap, which indicates that the regulation of the adaptive response of the plant is finely balanced between protection against aggressors and acquisition of benefits. The high level of complexity of the plant's capacity to respond to its biotic environment is likely to be the product of the co-evolutionary arms race between plants and their attackers. To understand the functioning of the complex defense signaling network in nature, molecular biologists and ecologists have joined forces to place molecular mechanisms of induced plant defenses in an ecological perspective. Here, we review recent findings in this rapidly expanding field of research.

\section{Molecular biology meets nature}

Studies on the mechanisms of plant defense responses usually consider individual plant-attacker interactions under a limited set of abiotic conditions. Because plant defense mechanisms have evolved during the co-evolutionary arms race between plants and their attackers and come with costs in addition to benefits $[1,10,12]$, insights into their significance for plant fitness should ideally come from ecological studies. Recently, a meta-analysis of plant performance during the interaction with multiple enemies and mutualists highlighted the enormous complexity of the plant's capacity to respond to other organisms in its environment [18]. In recent years, ecologists have embraced Arabidopsis thaliana, a member of the Brassicaceae, as a model plant [19-21]. Through a combined molecular genetics and ecological approach using Arabidopsis, important progress has been made in our understanding of how plants defend themselves against pathogens and insects from both a mechanistic and an ecological point of view. Although Arabidopsis is not an ideal ecological model plant, it can be a valuable stepping stone towards investigations of ecological model plants in the Brassicaceae family. In addition, non-brassicaceous 


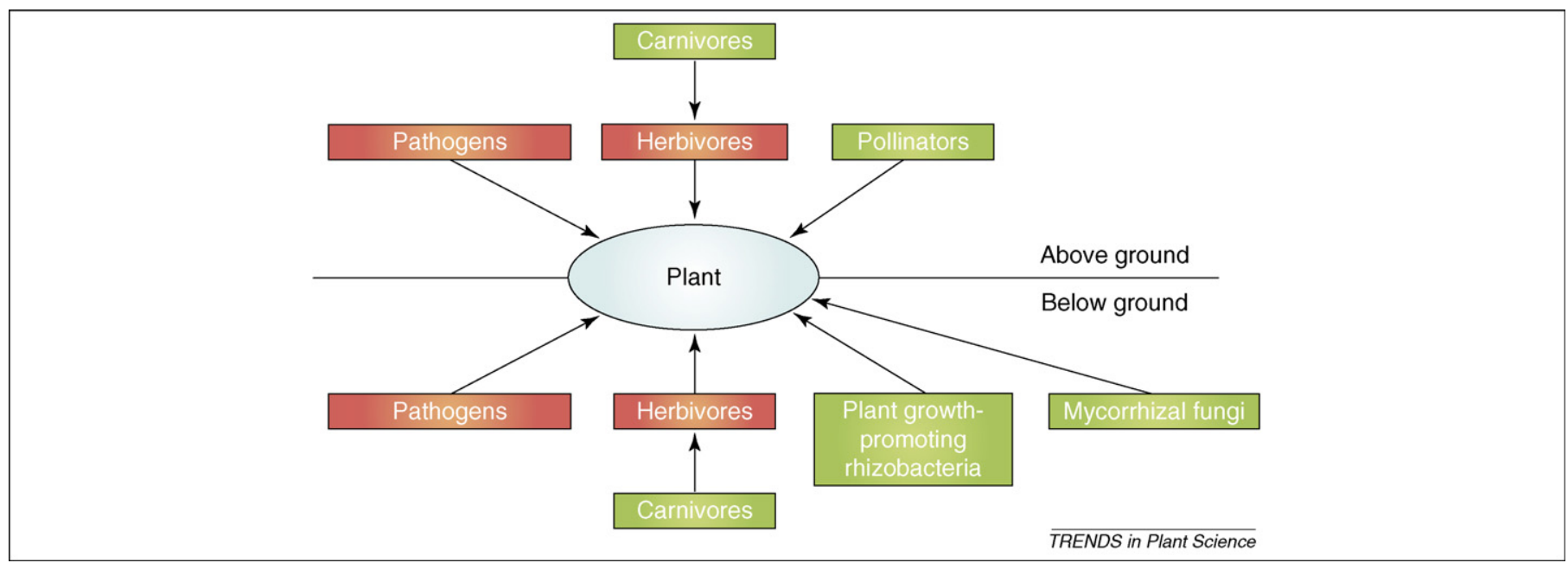

Figure 1. Plants as members of complex communities. Plants interact with attackers, such as microbial pathogens and herbivorous arthropods (red) and beneficia organisms, such as rhizobacteria, mycorrhizal fungi, carnivorous arthropods and pollinators (green). The signaling networks that are activated by the plant in response to its friends and foes overlap, indicating that the regulation of the adaptive response of the plant is finely balanced between protection against aggressors and acquisition of benefits.

plant species, such as the wild tobacco Nicotiana attenuata, have been developed into excellent models for studying molecular ecology of plant defense beyond the laboratory bench [22].

Although it is common knowledge that plants are members of complex communities and that individual characteristics, such as resistance to pathogens and insects, express natural variation, most studies that focus on the mechanisms of induced resistance concentrate on single plant-attacker combinations with 'digital' genotypes, such as knockout mutants and transgenics that overexpress individual genes. However, interactions with a single attacker modify the phenotype of the plant and, consequently, might affect interactions with other community members. Commonly, these phenomena are quantitatively variable within and between individuals. Unraveling the

\section{Box 1. Plant self defense}

In nature, plants interact with a range of organisms, some of which are harmful (e.g. pathogens or herbivorous insects), whereas others are beneficial (e.g. growth-promoting rhizobacteria, mycorrhizal fungi, predatory enemies of herbivores, and pollinators). Plants have evolved highly sophisticated strategies to counteract attackers and to accommodate mutualists (Figure I). The primary immune response has evolved to recognize common features of microbial pathogens that are referred to as pathogen-associated molecular patterns (PAMPs) [10,12]. PAMP-triggered immunity is part of the first line of defense and results in a basal level of resistance. During the coevolutionary arms race between plants and their microbial attackers, pathogens acquired the ability to suppress this first line of defense via the delivery of effector proteins. In turn, plants acquired $R$ proteins that recognize these attacker-specific effector proteins, resulting in a second line of defense called 'effector-triggered immunity' $[10,12]$. Ecologists have intensely debated the arms race between plants and herbivorous insects [1]. However, our understanding of the underlying mechanisms is relatively limited compared with the well-studied mechanisms involved in the arms race between pathogens and their host plants (but see [1]).

In addition to the immune response triggered following attackerspecific recognition, plants can activate another line of defense that is referred to as 'induced resistance'. Induced resistance often acts systemically throughout the plant and is characterized by a broad spectrum of effectiveness [80]. Induced resistance can be activated by microbial pathogens and insect herbivores, but also by beneficial microorganisms, such as mycorrhizal fungi and plant growth-promoting rhizobacteria $[7,9,11]$. Well-studied examples of induced resistance are pathogen-induced systemic acquired resistance (SAR) [23], rhizobacteria-mediated induced systemic resistance (ISR) [5] and herbivoreinduced direct defense $[11,81]$ and indirect defense $[6,21]$. Induced defense mechanisms involve the production of defensive compounds, such as pathogenesis-related (PR) proteins with antimicrobial activity [82], proteinase inhibitors that affect insect feeding [81], volatiles that attract parasitoids and predators of the herbivores that feed on the plant [6] and extrafloral nectar that arrests carnivorous arthropods on herbivore-infested plants [65]. The plant hormones salicylic acid (SA), jasmonic acid (JA) and ethylene (ET) are key players in the regulation of induced resistance. The molecular mechanisms underlying induced plant defense have been intensely studied in model plants, such as Arabidopsis. Now, ecologists are putting the laboratory facts to the test in ecological model plants in nature (e.g. $[22,65])$.
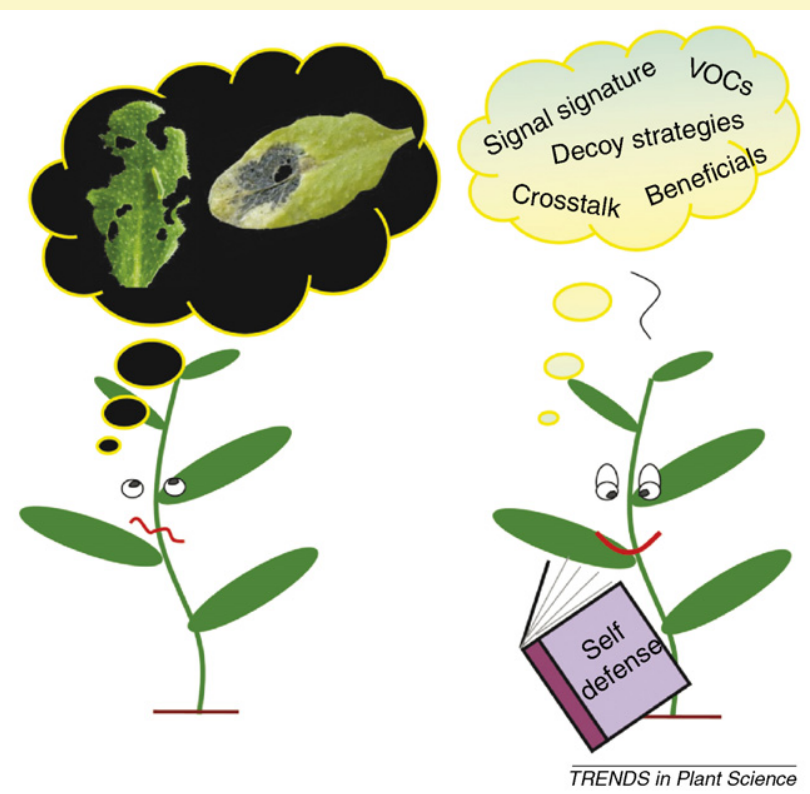

Figure I. Plant self defense. During evolution, plants acquired sophisticated defense mechanisms to counteract attack by microbial pathogens and herbivorous insects and to accommodate beneficial mutualists: VOCs= Volatile Organic Compounds, such as green leafy volatiles, terpenes, methyl salicylate and others. Cartoon by Marieke van Hulten. 
mechanisms and ecological functions of plants interacting with multiple species increases the complexity that investigators face. This complexity is both a challenge and a necessity as it is a first step towards unraveling the ecological relevance in terms of costs and benefits of the defensive mechanisms in a multitrophic environment. The detailed understanding of defense mechanisms and their induction by different attackers provides new methods for manipulative experiments that precisely alter the phenotype of the plant. This enables the investigation of the ecological consequences of changes in plant phenotype.

\section{Defense signaling networks}

The use of model plant systems, such as Arabidopsis, and various plant genomics approaches has expanded our understanding of the molecular mechanisms by which plants tailor their responses to parasites, herbivores and beneficial organisms. The plant hormones salicylic acid (SA), jasmonic acid (JA) and ethylene (ET) emerged as key players in the regulation of signaling networks involved in these responses [21,23-25]. Other plant hormones, including abscisic acid [26], brassinosteroids [27] and auxins [28], have also been reported to have a role in the immune response of the plant, but their significance is less well studied. SA-, JA- and ET-dependent pathways regulate defense responses that are differentially effective against specific types of attackers. Although there are exceptions [29], generally speaking, pathogens that require a living host to complete their life cycle (biotrophs) are more sensitive to SA-dependent defense responses, whereas pathogens that kill the host and feed on the content (necrotrophs) and herbivorous insects are affected by JA- and ET-dependent defenses [30,31]. In beneficial interactions, JA and other oxylipins also seem to have a dominant role in the response of the plant $[7,21,32]$.

The defense signaling pathways that are activated during the interactions of plants with herbivores, pathogens and beneficials show a high degree of overlap, which raises the question whether the activation of the respective defense responses leads to similarities in the spectrum of effectiveness and cross-resistance. Indeed, activation of JA- and ET-dependent induced systemic resistance (ISR) in Arabidopsis by beneficial rhizobacteria (Pseudomonas fluorescens) results in enhanced protection against predominantly necrotrophic pathogens that are sensitive to JA-dependent defenses; by contrast, induction of SAdependent systemic acquired resistance (SAR) by necrotizing pathogens is primarily effective against biotrophic pathogens that are deterred by SA-dependent defenses [33]. Furthermore, caterpillars of the herbivore Pieris rapae (small cabbage white butterfly), which stimulate the production of JA and ET in Arabidopsis, trigger a defense response that not only affects insect performance but also provides enhanced protection against the microbial pathogens Pseudomonas syringae, Xanthomonas campestris and Turnip crinkle virus [34]. Insight into the spectrum of effectiveness of the induced defense signaling pathways provides important information that will help to explain the ecological relevance of induced defense mechanisms in nature.

\section{Fine-tuning of defense}

A major focus in plant defense signaling research is to uncover key mechanisms by which plants tailor their responses to attackers and mutualists, and to investigate how plants cope with simultaneous interactions with multiple aggressors. The regulatory potential of the socalled 'signal signature' has an important role in the finetuning of the plant's defense response [35]. In Arabidopsis, the production of the defense signals SA, JA and ET has been shown to vary depending on the type of organism that interacts with the plant [13]. The quantity, composition and timing of this signal signature results in the activation of a specific set of genes that eventually shapes the outcome of the defense response that is triggered by the encountered attacker $[13,36]$. However, additional ways of regulation are also important. For example, the bacterial pathogen $P$. syringae, the necrotrophic fungus Alternaria brassicicola, the cell-content feeding thrips Frankliniella occidentalis and the chewing caterpillar $P$. rapae all stimulate JA biosynthesis and JA-responsive gene expression in Arabidopsis [13]. Yet, most JA-responsive genes that were activated by each attacker were specific for the plantattacker combination. Hence, the signal signature has an important primary role in the orchestration of the defense response of the plant, but other regulatory mechanisms, such as pathway crosstalk, or additional attackerinduced signals, eventually shape the highly complex attacker-specific defense response.

\section{Molecular players in pathway crosstalk}

There is ample evidence that SA, JA and ET pathways interact, either positively or negatively [37-39]. Global expression profiling of pathogen-infected Arabidopsis wild type and signaling mutants highlighted substantial crosstalk between the SA, JA and ET signaling pathways [40]. In some cases, the simultaneous activation of multiple defense pathways results in enhanced levels of induced resistance, whereas in other cases antagonistic effects on pathogen and insect resistance have been reported [38]. Several key regulatory proteins involved in pathway crosstalk have been identified. For instance, NPR1 (NON-EXPRESSER OF $P R-1)$ and glutaredoxin have an important role in the antagonistic effect of SA on JA-responsive gene expression $[41,42]$. The transcription factor WRKY70 was shown to function as an activator of SA-responsive genes and a repressor of JA-inducible genes, thereby functioning as a molecular switch between both pathways [43]. Furthermore, the transcription factors ERF1 (ETHYLENE RESPONSE FACTOR1) and MYC2 were found to function as important signaling nodes that integrate signals from the JA and ET pathways and differentially activate JA-dependent defense genes [44,45]. In Arabidopsis, the regulatory proteins NPR1, WRKY70, ERF1 and MYC2 have an important role in shaping the outcome of the plant's defense response. However, their ecological importance has yet to be elucidated.

\section{Decoy of plant defenses}

Crosstalk between defense pathways is thought to provide the plant with a powerful regulatory potential, which helps it to prioritize and 'decide' which defensive strategy to 
follow, depending on the type of attacker. Yet, it seems that attackers and beneficial organisms have also evolved to manipulate plants for their own benefit by shutting down induced defenses by modulating the signaling network $[3,46,47]$. An example is the response of Arabidopsis to silverleaf whitefly (Bemisia tabaci) nymphs. The nymphs of this phloem-feeding insect sabotage effective JA-dependent host defenses by activating the antagonistic SA signaling pathway [48].

Pathogens also suppress effective defenses using virulence factors that modulate host defenses. A well-studied example is the plant $-P$. syringae interaction. Several virulence factors of this bacterial pathogen have been demonstrated to suppress host defense responses [49]. One of these virulence factors is the phytotoxin coronatine, which functions as ajasmonate analog [49]. During the interaction with susceptible Arabidopsis plants, coronatine suppresses effectual SA-dependent defenses, thereby promoting susceptibility of the plant to this pathogen [50-52]. Interestingly, the degree of interplay between the SA- and JA-dependent defenses in this plant-pathogen interaction differs among Arabidopsis accessions [53], which suggests intraspecific variability in how these defense pathways cross-communicate. In an ecological context, this natural variation in pathway crosstalk might be instrumental in avoiding the decoy strategies that are employed by the plant's enemies.

\section{Priming for enhanced defense}

Differential signal signatures, pathway crosstalk and attacker-mediated suppression of host defense signaling are major molecular mechanisms by which the defense response of the plant is shaped. Priming for enhanced defense adds another layer of complexity to the way by which plants can adapt to their biotic environment. The primed state can be induced biologically by beneficial rhizobacteria [14], mycorrhizal fungi [7], pathogens [54] and insect herbivores [34,55], but also chemically, for example, by exogenous application of low doses of SA [56], JA [57] or $\beta$-aminobutyric acid [58]. In primed plants, defense responses are not activated directly by the priming agent, but are accelerated following perception of biotic or abiotic stress signals, resulting in an enhanced level of resistance $[59,60]$. Through the study of the costs and benefits of priming in Arabidopsis, it was recently shown that the fitness costs of priming are lower than those of constitutively activated defenses, such as those expressed in the constitutive SAR-expressing mutant cpr1 [61]. Intriguingly, the fitness benefits of priming outweighed its costs under pathogen pressure, which suggests that priming functions as an ecological adaptation of the plant to respond faster to its hostile environment.

Priming by airborne signals, such as volatile organic compounds (VOCs) produced following insect herbivory, is a major topic in molecular ecological research on plantherbivore and plant-plant interactions [62]. Engelberth and coworkers [55] demonstrated that the green leafy volatiles produced by corn plants after insect feeding prime (rather than directly activate) neighboring plants for enhanced defense against herbivory. In a laboratory study with maize, VOCs were similarly demonstrated to prime neighboring plants for enhanced direct and indirect defense, which resulted in a reduced performance of caterpillars of the Egyptian cotton leafworm Spodoptera littoralis (direct defense) and improved the attractiveness to the parasitoid wasps Cotesia marginiventris that feed on the insect herbivores (indirect defense) [63]. Also in the field, herbivory-induced VOCs have been demonstrated to prime nearby plants for enhanced direct and indirect defense responses [64], indicating that priming has a role in plant defense under ecologically realistic conditions. Recently, another demonstration of VOC-induced priming in nature was provided by Heil and Silva Bueno [65]. They demonstrated that VOCs released by beetle-infested 'emitter' leaves of lima bean plants (Phaseolus lunatus) growing in their natural habitat primed nearby 'receiver' leaves for enhanced secretion of extrafloral nectar, resulting in prolonged visitation by predatory arthropods. Together, these examples highlight the ecological benefits of the priming phenomenon for plants growing under field conditions.

\section{Molecular ecology: beyond Arabidopsis}

Ecologists have provided ample data on plant-microbe and plant-insect interactions. For instance, mycorrhizae have been shown to affect interactions between plants and pollinators, herbivorous insects and their parasitic wasps [66-68]. Conversely, herbivory can influence mycorrhizal colonization of plants. However, in most cases, the underlying mechanisms, in terms of signal transduction and gene expression, have yet to be investigated. Research on Arabidopsis has contributed to our understanding of molecular mechanisms of plant-microbe and plant-insect interactions [21,69]. In addition to the availability of the full genome sequence, the ample availability of mutant, transgenic and reporter lines provides researchers with valuable genetic tools to address specific questions on the mechanisms of plant responses to attackers and beneficial organisms. Although many parasitic and beneficial microbes and insects used in conjunction with Arabidopsis have limited relevance under field conditions [70], this model system provides a stepping stone to other brassicaceous plants. For instance, the sequence identity in coding regions of genes is $>85 \%$ for Arabidopsis and Brassica [71] and 70-mer oligo-microarrays developed for Arabidopsis have been successfully used to investigate global gene expression in Brassica [72,73]. This high level of similarity has opened the way for comparative genomics of Brassicaceae species, which have already resulted in excellent tools for the study of ecologically relevant traits, such as tolerance of salt, drought or flooding [74]. The knowledge gained for Arabidopsis will facilitate molecular ecological research in wild and cultivated Brassica species that have already been well studied by ecologists [75,76]. Moreover, rapid progress is being made with the development of molecular tools for other ecological model plants, such as $N$. attenuata and Solanum nigrum (black nightshade) $[62,77]$. By cloning genes and developing methodologies to genetically modify these ecological model plants, interesting experiments can be carried out to put laboratory findings to the test under field conditions in the native habitats. Such experiments show, for example, that silencing the JA cascade turns $N$. attenuata into a suitable host plant for herbivores that are otherwise never recorded on 
this plant [22]. Moreover, the attack by one herbivore was shown to influence the success of attack by a second herbivore species that was mediated by changes in direct and indirect defenses [78,79]. These examples nicely demonstrate that molecular ecological studies provide powerful tools to investigate how defense mechanisms identified in model plant species can function in nature.

\section{Concluding remarks and future perspectives}

The reductionist approach taken in plant defense research has resulted in profound insight into the complexity of plant defense responses. The wealth of Arabidopsis mutants that are available has resulted in important progress in our understanding of how plants interact with individual microbe or arthropod species. This knowledge is now being further developed to address interactions between Arabidopsis and two or more species, including pathogenic and beneficial microbes and herbivorous and carnivorous insects. From the other end of the spectrum, ecologists have addressed the interactions between plants and their community members, thus providing insights into the effects of plant defense-related traits on organisms at different trophic levels, such as herbivores and carnivores. By bringing together these approaches and investigating plant responses from the molecular genetic level to individual species interactions and community ecology, the new field of molecular ecology or 'ecogenomics' is developing rapidly. At present, this is done by a 'digital' approach in which individual genes are being knocked out or overexpressed, followed by an assessment of the resulting effects on interactions between the plant and microbes or insects. Although this is an important step that provides interesting new insights, this is only a first step. In nature, plant defense traits are often polygenic and vary quantitatively among genotypes. Hence, the real challenge is to explore this natural variation because it is a fantastic reservoir of natural adaptive mechanisms that have evolved to respond to biotic stressors and that, to date, have remained unknown or poorly understood. To achieve this, collaboration between ecologists and molecular geneticists is vital to characterize natural variation within and among natural populations and to develop genotypes that express quantitative variation in particular characteristics. This line of investigation, in conjunction with the effects on individual community members or on the fitness of plants within complex communities, will provide new information on the costs and benefits of plant defenses and will potentially be used for the development of novel strategies for crop protection.

\section{Acknowledgements}

We apologize to all our colleagues whose work could not be reviewed here because of space limitations. Our laboratories have been financially supported (grants $811.36 .004,865.03 .002$ and 865.04 .002 ) by the Earth and Life Sciences Foundation (ALW), which is subsidized by the Netherlands Organization for Scientific Research (NWO).

\section{References}

1 Schoonhoven, L.M. et al., eds (2005) Insect-Plant Biology, Oxford University Press

2 Agrios, G.N., ed. (2005) Plant Pathology, Elsevier Academic Press

3 Harrison, M.J. (2005) Signaling in the arbuscular mycorrhizal symbiosis. Annu. Rev. Microbiol. 59, 19-42
4 Holub, E.B. (2006) Evolution of symbioses between plants and filamentous parasites. Curr. Opin. Plant Biol. 9, 397-405

5 Van Loon, L.C. et al. (1998) Systemic resistance induced by rhizosphere bacteria. Annu. Rev. Phytopathol. 36, 453-483

6 Dicke, M. et al. (2003) Plants talk, but are they deaf? Trends Plant Sci. $8,403-405$

7 Pozo, M.J. et al. (2004) Jasmonates - signals in plant-microbe interactions. J. Plant Growth Regul. 23, 211-222

8 Kessler, D. and Baldwin, I.T. (2007) Making sense of nectar scents: the effects of nectar secondary metabolites on floral visitors of Nicotiana attenuata. Plant J. 49, 840-854

9 Dicke, M. and Hilker, M. (2003) Induced plant defences: from molecular biology to evolutionary ecology. Basic Appl. Ecol. 4, 3-14

10 Chisholm, S.T. et al. (2006) Host-microbe interactions: shaping the evolution of the plant immune response. Cell 124, 803-814

11 Kessler, A. and Baldwin, I.T. (2002) Plant responses to insect herbivory: The emerging molecular analysis. Annu. Rev. Plant Biol. $53,299-328$

12 Jones, J.D.G. and Dangl, J.L. (2006) The plant immune system. Nature $444,323-329$

13 De Vos, M. et al. (2005) Signal signature and transcriptome changes of Arabidopsis during pathogen and insect attack. Mol. Plant-Microbe Interact. 18, 923-937

14 Verhagen, B.W. et al. (2004) The transcriptome of rhizobacteriainduced systemic resistance in Arabidopsis. Mol. Plant-Microbe Interact. 17, 895-908

15 Kempema, L.A. et al. (2007) Arabidopsis transcriptome changes in response to phloem-feeding silverleaf whitefly nymphs. Similarities and distinctions in responses to aphids. Plant Physiol. 143, 849-865

16 Reymond, P. et al. (2004) A conserved transcriptional pattern in response to a specialist and a generalist herbivore. Plant Cell 16, 3132-3147

17 Sanchez, L. et al. (2005) Pseudomonas fluorescens and Glomus mosseae trigger DMI3-dependent activation of genes related to a signal transduction pathway in roots of Medicago truncatula. Plant Physiol. 139, 1065-1077

18 Morris, W.F. et al. (2007) Direct and interactive effects of enemies and mutualists on plant performance: A meta-analysis. Ecology 88, 10211029

19 Holub, E.B. (2001) The Arms race is ancient history in Arabidopsis, the wildflower. Nat. Rev. Genet. 2, 516-527

20 Mitchell-Olds, T. (2001) Arabidopsis thaliana and its wild relatives: A model system for ecology and evolution. Trends Ecol. Evol. 16, 693-700

21 Van Poecke, R.M.P. and Dicke, M. (2004) Indirect defence of plants against herbivores: using Arabidopsis thaliana as a model plant. Plant Biol. 6, 387-401

22 Kessler, A. et al. (2004) Silencing the jasmonate cascade: induced plant defenses and insect populations. Science 305, 665-668

23 Durrant, W.E. and Dong, X. (2004) Systemic acquired resistance. Annu. Rev. Phytopathol. 42, 185-209

24 Grant, M. and Lamb, C. (2006) Systemic immunity. Curr. Opin. Plant Biol. 9, 414-420

25 Von Dahl, C.C. and Baldwin, I.T. (2007) Deciphering the role of ethylene in plant-herbivore interactions. J. Plant Growth Regul. 26, 201-209

26 Mauch-Mani, B. and Mauch, F. (2005) The role of abscisic acid in plantpathogen interactions. Curr. Opin. Plant Biol. 8, 409-414

27 Nakashita, H. et al. (2003) Brassinosteroid functions in a broad range of disease resistance in tobacco and rice. Plant J. 33, 887898

28 Navarro, L. et al. (2006) A plant miRNA contributes to antibacterial resistance by repressing auxin signaling. Science $312,436-439$

29 Thaler, J.S. et al. (2004) The role of the jasmonate response in plant susceptibility to diverse pathogens with a range of lifestyles. Plant Physiol. 135, 530-538

30 Glazebrook, J. (2005) Contrasting mechanisms of defense against biotrophic and necrotrophic pathogens. Annu. Rev. Phytopathol. 43, 205-227

31 Thomma, B.P.H.J. et al. (2001) The complexity of disease signaling in Arabidopsis. Curr. Opin. Immunol. 13, 63-68

32 Hause, B. and Fester, T. (2005) Molecular and cell biology of arbuscular mycorrhizal symbiosis. Planta 221, 184-196 
33 Ton, J. et al. (2002) Differential effectiveness of salicylate-dependent and jasmonate/ethylene-dependent induced resistance in Arabidopsis. Mol. Plant-Microbe Interact. 15, 27-34

34 De Vos, M. et al. (2006) Herbivore-induced resistance against microbial pathogens in Arabidopsis. Plant Physiol. 142, 352-363

35 Reymond, P. and Farmer, E.E. (1998) Jasmonate and salicylate as global signals for defense gene expression. Curr. Opin. Plant Biol. 1, 404-411

36 Mur, L.A.J. et al. (2006) The outcomes of concentration-specific interactions between salicylate and jasmonate signaling include synergy, antagonism, and oxidative stress leading to cell death. Plant Physiol. 140, 249-262

37 Beckers, G.J.M. and Spoel, S.H. (2006) Fine-tuning plant defence signalling: salicylate versus jasmonate. Plant Biol. 8, 1-10

38 Bostock, R.M. (2005) Signal crosstalk and induced resistance: straddling the line between cost and benefit. Annu. Rev. Phytopathol. 43, 545580

39 Rojo, E. et al. (2003) Interactions between signaling compounds involved in plant defense. J. Plant Growth Regul. 22, 82-98

40 Glazebrook, J. et al. (2003) Topology of the network integrating salicylate and jasmonate signal transduction derived from global expression phenotyping. Plant J. 34, 217-228

41 Ndamukong, I. et al. (2007) SA-inducible Arabidopsis glutaredoxin interacts with TGA factors and suppresses JA-responsive PDF1.2 transcription. Plant J. 50, 128-139

42 Spoel, S.H. et al. (2003) NPR1 modulates cross-talk between salicylateand jasmonate-dependent defense pathways through a novel function in the cytosol. Plant Cell 15, 760-770

$43 \mathrm{Li}$, J. et al. (2004) The WRKY70 transcription factor: a node of convergence for jasmonate-mediated and salicylate-mediated signals in plant defense. Plant Cell 16, 319-331

44 Lorenzo, O. et al. (2004) JASMONATE-INSENSITIVE1 encodes a MYC transcription factor essential to discriminate between different jasmonate-regulated defense responses in Arabidopsis. Plant Cell 16, $1938-1950$

45 Lorenzo, O. et al. (2003) ETHYLENE RESPONSE FACTOR1 integrates signals from ethylene and jasmonate pathways in plant defense. Plant Cell 15, 165-178

$46 \mathrm{Kahl}$, J. et al. (2000) Herbivore-induced ethylene suppresses a direct defense but not a putative indirect defense against an adapted herbivore. Planta 210, 336-342

47 Musser, R.O. et al. (2002) Herbivory: Caterpillar saliva beats plant defences - a new weapon emerges in the evolutionary arms race between plants and herbivores. Nature 416, 599-600

48 Zarate, S.I. et al. (2007) Silverleaf whitefly induces salicylic acid defenses and suppresses effectual jasmonic acid defenses. Plant Physiol. 143, 866-875

49 Nomura, K. et al. (2005) Suppression of host defense in compatible plant-Pseudomonas syringae interactions. Curr. Opin. Plant Biol. 8, 361-368

50 Brooks, D.M. et al. (2005) The Pseudomonas syringae phytotoxin coronatine promotes virulence by overcoming salicylic aciddependent defences in Arabidopsis thaliana. Mol. Plant Pathol. 6, 629-639

51 Laurie-Berry, N. et al. (2006) The Arabidopsis thaliana JASMONATE INSENSITIVE 1 gene is required for suppression of salicylic aciddependent defenses during infection by Pseudomonas syringae. Mol. Plant-Microbe Interact. 19, 789-800

52 Cui, J. et al. (2005) Pseudomonas syringae manipulates systemic plant defenses against pathogens and herbivores. Proc. Natl. Acad. Sci. U.S.A. $102,1791-1796$

53 Traw, M.B. et al. (2003) Negative cross-talk between salicylate- and jasmonate-mediated pathways in the Wassilewskija ecotype of Arabidopsis thaliana. Mol. Ecol. 12, 1125-1135

54 Cameron, R.K. et al. (1999) Accumulation of salicylic acid and $P R$ gene transcripts in relation to the systemic acquired resistance (SAR) response by Pseudomonas syringae pv. tomato in Arabidopsis. Physiol. Mol. Plant Pathol. 55, 121-130

55 Engelberth, J.et al. (2004) Airborne signals prime plants against insect herbivore attack. Proc. Natl. Acad. Sci. U. S. A. 101, 1781-1785
56 Mur, L.A.J. et al. (1996) Salicylic acid potentiates defence gene expression in tissue exhibiting acquired resistance to pathogen attack. Plant J. 9, 559-571

57 Kauss, H. et al. (1994) Pretreatment of parsley (Petroselinum crispum L.) suspension cultures with methyl jasmonate enhances elicitation of activated oxygen species. Plant Physiol. 105, 89-104

58 Ton, J. et al. (2005) Dissecting the $\beta$-aminobutyric acid-induced priming phenomenon in Arabidopsis. Plant Cell 17, 987-999

59 Conrath, U. et al. (2006) Priming: getting ready for battle. Mol. PlantMicrobe Interact. 19, 1062-1071

60 Conrath, U. et al. (2002) Priming in plant-pathogen interactions. Trends Plant Sci. 7, 210-216

61 Van Hulten, M. et al. (2006) Costs and benefits of priming for defense in Arabidopsis. Proc. Natl. Acad. Sci. U. S. A. 103, 5602-5607

62 Baldwin, I.T. et al. (2006) Volatile signaling in plant-plant interactions: "Talking trees" in the genomics era. Science $311,812-815$

63 Ton, J. et al. (2007) Priming by air-borne signals boosts direct and indirect resistance in maize. Plant J. 49, 16-26

64 Kessler, A. et al. (2006) Priming of plant defense responses in nature by airborne signaling between Artemisia tridentata and Nicotiana attenuata. Oecologia 148, 280-292

65 Heil, M. and Silva Bueno, J.C. (2007) Within-plant signaling by volatiles leads to induction and priming of an indirect plant defense in nature. Proc. Natl. Acad. Sci. U. S. A. 104, 5467-5472

66 Gange, A.C. and Smith, A.K. (2005) Arbuscular mycorrhizal fungi influence visitation rates of pollinating insects. Ecol. Entomol. 30, 600-606

67 Goverde, M. et al. (2000) Arbuscular mycorrhizal fungi influence life history traits of a lepidopteran herbivore. Oecologia 125, 362-369

68 Guerrieri, E. et al. (2004) Do interactions between plant roots and the rhizosphere affect parasitoid behaviour? Ecol. Entomol. 29, 753-756

69 Pieterse, C.M.J. et al. (2002) Signalling in rhizobacteria-induced systemic resistance in Arabidopsis thaliana. Plant Biol. 4, 535-544

70 Harvey, J.A. et al. (2007) Nutritional suitability and ecological relevance of Arabidopsis thaliana and Brassica oleracea as foodplants for the cabbage butterfly, Pieris rapae. Plant Ecol. 189, $117-126$

71 Cavell, A.C. et al. (1998) Collinearity between a 30-centimorgan segment of Arabidopsis thaliana chromosome 4 and duplicated regions within the Brassica napus genome. Genome 41, 62-69

72 Lee, H-S. et al. (2004) Sensitivity of 70-mer oligonucleotides and cDNAs for microarray analysis of gene expression in Arabidopsis and its related species. Plant Biotechnol. J. 2, 45-57

73 Broekgaarden, C. et al. (2007) Genotypic variation in genome-wide transcription profiles induced by insect feeding: Brassica oleraceaPieris rapae interactions. BMC Genomics 8, 239

74 Schranz, M.E. et al. (2007) Comparative genomics in the Brassicaceae: a family-wide perspective. Curr. Opin. Plant Biol. 10, 168-175

75 Harvey, J.A. et al. (2003) Interactions over four trophic levels: foodplant quality affects development of a hyperparasitoid as mediated through a herbivore and its primary parasitoid. J. Anim. Ecol. 72, 520-531

76 Moyes, C.L. et al. (2000) Glucosinolates and differential herbivory in wild populations of Brassica oleracea. J. Chem. Ecol. 26, 2625-2641

77 Schmidt, D.D. et al. (2004) Solanum nigrum: A model ecological expression system and its tools. Mol. Ecol. 13, 981-995

78 Kessler, A. and Baldwin, I.T. (2004) Herbivore-induced plant vaccination. Part I. The orchestration of plant defenses in nature and their fitness consequences in the wild tobacco Nicotiana attenuata. Plant J. 38, 639-649

79 Voelckel, C. and Baldwin, I.T. (2004) Herbivore-induced plant vaccination. Part II. Array-studies reveal the transience of herbivore-specific transcriptional imprints and a distinct imprint from stress combinations. Plant J. 38, 650-663

80 Walters, D. et al. (2007) Induced Resistance for Plant Defence: A Sustainable Approach to Crop Protection, Blackwell

81 Howe, G.A. (2004) Jasmonates as signals in the wound response. J. Plant Growth Regul. 23, 223-237

82 Van Loon, L.C. et al. (2006) Significance of inducible defense-related proteins in infected plants. Annu. Rev. Phytopathol. 44, 135-162 